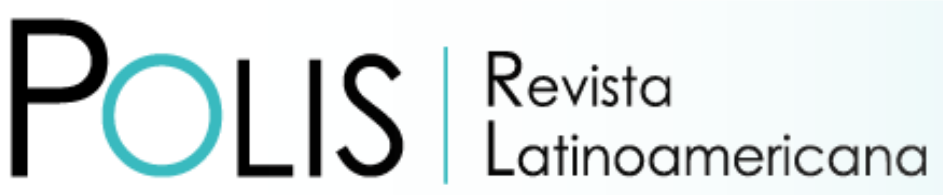

V20 | N59 | 2021

DOI:10.32735/S0718-6568/2021-N59-1587

\title{
Página en blanco: discursos de resistencia para reconocer y documentar la violencia contra la mujer en el ambiente académico
}

\author{
Angélica María Hernández-Ramírez \\ Universidad Veracruzana, Xalapa, México \\ Email: angehernandez@uv.mx
}

Recibido:27.03.2020

Aceptado: 04.01.2021

\begin{abstract}
Resumen: Mientras las mujeres experimentan diferentes formas de violencia, los perpetradores usan las prácticas y comportamientos de las instituciones para controlar a las mujeres mediante coerción, fuerza física o silencio. Este estudio revisa y analiza el marco legal y los discursos públicos asociados a los informes de quejas dirigidos a la Universidad Veracruzana. México posee un marco legal para promover los derechos de las mujeres y la universidad posee un marco regulatorio para apoyar el bienestar de las mujeres en el ambiente académico. Pero, existe un discurso de resistencia basado en la normalización, desvío de la atención y prácticas que legitiman la violencia contra las mujeres en el ambiente académico. La falta de indicadores de violencia contra la mujer, como página en blanco, son más que la ausencia de información en un documento; anticipa procesos ocultos y de incomprensión asociado a las experiencias que enfrentan las mujeres en el ambiente académico.
\end{abstract}

Palabras clave: Impunidad; inequidad de género; poder; Universidad Veracruzana; violencia contra las mujeres.

\section{Blank Page: Resistance Discourses to Identify and Document Violence against Women in Academia}

\begin{abstract}
Whereas women experience violence in many different ways, perpetrators use institutional practices and behaviors to exercise control over women through coercion, physical force, or silencing. This study reviews and analysis the legal framework and public discourses associated with reports of complaints addressed to the University of Veracruz. Mexico has a legal framework to promote women rights, whereas the university has a regulatory framework to support women's well-being in the academic environment. Nonetheless, there is a discourse of resistance that uses strategies of naturalization, attention diversion, and practices that legitimate violence against women in the academic environment. The lack of indicators of violence against woman, like a blank page, it is more than the absence of information in a document; it anticipates hidden and misunderstood processes regarding experiences that women face in the academic environment.
\end{abstract}

Keywords: Gender inequality; Impunity; University of Veracruz; Violence against women. 


\title{
Página em branco: discursos de resistência para reconhecer e documentar a violência contra as mulheres no meio acadêmico
}

\begin{abstract}
Resumo: Enquanto as mulheres experimentam diferentes formas de violência, os autores usam as práticas e comportamentos das instituições para controlar as mulheres por meio de coerção, força física ou silêncio. Este estudo analisa a estrutura legal e os discursos públicos associados aos relatórios de reclamações encaminhados à Universidade Veracruzana. 0 México possui uma estrutura legal para promover os direitos das mulheres e a universidade possui uma estrutura reguladora para apoiar o bem-estar das mulheres no ambiente acadêmico. Porém, há um discurso de resistência baseado na normalização, desvio de atenção e práticas que legitimam a violência contra a mulher no ambiente acadêmico. A falta de indicadores de violência contra as mulheres, como uma página em branco, é mais do que a ausência de informações em um documento; antecipa processos ocultos e mal-entendidos associados às experiências que as mulheres enfrentam no ambiente acadêmico.
\end{abstract}

Palavras-chave: Impunidade; desigualdade de gênero; poder; Universidade de Veracruz; violência contra as mulheres.

\section{Como citar este artículo:}

Hernández-Ramírez, A. (2021). Página en blanco: discursos de resistencia para reconocer y documentar la violencia contra la mujer en el ambiente académico. Polis Revista Latinoamericana, 20 (59), 185-202. doi: http://dx.doi.org/10.32735/S0718-6568/2021-N59-1587

\section{Introducción}

La violencia se entiende como el empleo de la fuerza física o coerción que se ejerce sobre un individuo y/o colectivo para que realice una acción contraria a su voluntad, libertad y/o juicio (Tedeschi y Felson, 1994). Por su parte, la denuncia constituye una vía por la cual los individuos y/o colectivos informan sobre hechos presuntamente delictivos a fin de proceder con una investigación, atribuir responsabilidades y obtener una resolución por parte de la autoridad competente (Tedeschi y Felson, 1994; Barreto, 2017). No obstante, la violencia puede nutrirse de acciones $u$ omisiones por parte de las autoridades que limitan, entorpecen y niegan el respeto al ejercicio pleno de los derechos fundamentales de los individuos y/o colectivos (Cantalupo, 2012; Mingo y Moreno, 2015).

Desde la perspectiva de género, la violencia contra la mujer ha mostrado tener una gran variedad de formas y suele ser poco reconocida, definida y examinada (García-Moreno et al., 2014). Por ello, se requiere revisar las normas, actitudes y creencias que justifican la violencia contra la mujer y que refuerzan la noción de dominio masculino en contextos reales de inequidad de género. Dicha inequidad de género se manifiesta cuando hay una evidente distribución desigual del poder entre mujeres y hombres, el cual llega ser aceptado y promovido a nivel social, cultural e institucional (van Roosmalen y McDaniel, 1998; Jordan, Combs y Smith, 2014; Hernández-Ramírez, 2017). La revisión del tema de violencia contra la mujer en distintos escenarios es clave para entender el fenómeno en sus múltiples facetas y contextos con la finalidad de afrontarlo, prevenirlo y erradicarlo. 
A nivel mundial se manifiestan y ejercen distintas formas de violencia contra la mujer, lo que ha permitido posicionar a este tema en la agenda política internacional y nacional. En el ámbito internacional destaca la Declaración sobre la eliminación de la violencia contra la mujer que fue aprobada por la Asamblea General de las Naciones Unidas en 1993, La Convención Interamericana para prevenir, sancionar y erradicar la Violencia contra la mujer (Convención Belém do Pará) celebrada en 1994 y la Cuarta Conferencia Mundial sobre la Mujer celebrada en Beijing en 1995. México se ha adherido a estas declaraciones y tratados en un esfuerzo por reconocer a la violencia contra las mujeres como un problema prioritario que atender. No obstante, existe un discurso de resistencia que atenta con situar el problema en su contexto social e institucional. Dicho discurso de resistencia incluye la normalización de la violencia contra la mujer, el desvío de la atención para la atribución de responsabilidades, así como un discurso asociado a una praxis que legitima la violencia y tiende a distorsionar este tipo de actos perpetuados (Berns, 2001; García-Moreno et al., 2014). Aunado a esto, el discurso de resistencia se utiliza como herramienta retórica para distraer la atención y trivializar el problema (Cantalupo, 2012; Mingo y Moreno, 2015; Barreto, 2017).

Con base en lo anterior, el objetivo principal de este estudio fue reconocer los discursos de resistencia que atentan con reconocer y documentar la violencia contra la mujer en la Universidad Veracruzana. Los objetivos particulares de este estudio fueron; 1) reconocer el tema de violencia contra la mujer desde el marco legislativo nacional, estatal e institucional como elementos clave que permite reconocer, nombrar, prevenir y atender el problema por parte de la autoridad competente y 2) identificar los discursos de resistencia que atentan con situar el problema de violencia contra la mujer en la institución. Específicamente; i) la normalización de la violencia contra la mujer, ii) el desvío de la atención para la atribución de responsabilidades y iii) los discursos y las prácticas que legitiman la violencia, fueron los elementos que se utilizaron para identificar los discursos de resistencia empleados en la institución.

Las Instituciones de Educación Superior constituye espacios privilegiados que de alguna $u$ otra forma legitiman los aprendizajes, habilidades, valores, actitudes, comportamientos y experiencias que vive la comunidad universitaria. De ahí su importancia en reconocer este tipo de violencia en el ambiente académico, ya que son centros formativos para la construcción de sociedades más justas e incluyentes. La Universidad Veracruzana se utilizó como estudio de caso, ya que es sectorizada por lo que se distribuye a lo largo del estado de Veracruz.

\section{La violencia contra la mujer en las Instifuciones de Educación Superior (IES)}

La normalización de la violencia contra la mujer en las IES ocurre a través de un patrón de comportamiento arraigado en las normas institucionales y en las prácticas cotidianas que refuerzan las jerarquías de privilegios y de desigualdad en el ejercicio del poder (Jordan, Combs y Smith, 2014; Barreto, 2017; Hernández-Ramírez, 2017). El desvío de la atención se presenta cuando no se sitúa y nombra claramente el problema en su contexto y en su lugar, se omiten indicadores de género a reportary se oculta/protege la identidad de los infractores 
(Mingo y Moreno, 2015). Por otro lado, la deficiencia en los procesos de investigación e impunidad son algunos de los factores clave que legitiman la violencia y distorsionan los actos de violencia cometidos en las instituciones (Berns, 2001; Jordan, Combs y Smith, 2014; Mingo y Moreno, 2015; Hernández-Ramírez, 2017). En particular, la violencia no-física y/o no-sexual constituye una modalidad de violencia común en el ambiente académico, el cual suele ser sutil, por lo que es difícil de tratar, reconocer y abordar (van Roosmalen y McDaniel, 1998; Huerta et al., 2006; Jordan, Combs y Smith, 2014; Hernández-Ramírez, 2017). No obstante, esta modalidad de violencia suele ser acumulativa e impacta negativamente la salud física, emocional y psicológica de los individuos afectados, el cual presenta una alta incidencia y prevalencia en la población femenina (DeKeseredy, 2000; Huerta et al., 2006; Jordan, Combs y Smith, 2014). El costo de esta modalidad de violencia a nivel institucional incluye altos índices de deserción en clases/cursos, retraso en los tiempos de titulación, cambio de carreras e incluso deserción en los estudios de los individuos afectados (Huerta et al., 2006; Mengo y Black, 2015).

En México, la violencia hacia las mujeres universitarias como problemática de investigación y de intervención ha sido poco abordado, por lo que ha tenido que encontrar respuestas en la denuncia pública (i.e., lucha por el reconocimiento; Mingo y Moreno, 2015; Barreto, 2017). Cabe mencionar que a nivel nacional no se tienen cifras generales o claras asociadas a este tipo de violencia. Como consecuencia de ello, las experiencias de vida y las necesidades de estas mujeres se han invisibilizado y perdido en el encubrimiento e impunidad. Por ello, en esta investigación se plantea el reconocer al tema de violencia contra la mujer y el discurso de resistencia que atenta con situar a este tipo de violencia en las IES. Para ello, se utiliza a la Universidad Veracruzana como estudio de caso que permite explorar y ejemplificar los avances y retos asociados con esta problemática en el país. Dicha problemática puede presentarse en otras IES localizadas en contextos latinoamericanos en los cuales prevalece el sentido de dominio masculino a nivel institucional. La Universidad Veracruzana sirve como modelo ideal para estudiar este tipo de problemas dado que se encuentra sectorizada en cinco regiones y cuatro sedes distribuidas a lo largo del estado de Veracruz. El estado de Veracruz se ubica en los primeros diez lugares con altas tasas de incidencia delictiva de 2010 a 2018, mientras que ocupó el primer lugar a nivel nacional en 2016 y 2017 (INEGI, 2018). Paralelamente, el Estado de Veracruz se encuentra por encima de la media (94.8\%) en delitos no denunciados (Semáforo de cifra negra; INEGI, 2019). A pesar de la alta tasa de incidencia delictiva con y sin denuncia formal, Veracruz se posiciona en el vigésimo tercer lugar (de un total de 32 estados en la República Mexicana) de entidades que poseen oficinas de derechos humanos por cada millón de habitantes a nivel nacional (INEGI, 2014). Aunado a esto, en Veracruz se presenta un porcentaje similar de delitos del fuero común y federal a nivel gubernamental (49\% policía estatal y $51 \%$ policía municipal; INEGI, 2015). En lo que respecta a violencia de género, se sabe que en el periodo comprendido del año 2000 a 2015 se reportaron 498 delitos catalogados como homicidio doloso contra mujeres y 96 delitos de feminicidio, lo que representó un incremento de casos de violencia contra la mujer del 33\% con respecto al reportado en octubre del año 2015 (Informe, 2016, 2017). Con base en los antecedentes e informes presentados por el Gobierno del Estado de Veracruz para el 
año 2016 y 2017, la Secretaría de Gobernación dictaminó que persiste en la entidad la situación de agravio comparado, asociado a los derechos humanos (restringidos) de las mujeres que está claramente contemplado en el Código Penal del Estado de Veracruz, por lo que persiste la falta de implementación correcta de la NOM-046-SSA2-20051 (SEGOB, 2016, 2017).

\section{Metodología}

La presente investigación parte de una revisión, análisis y reflexión relacionado con el tema de violencia contra la mujer desde dos perspectivas: 1) El marco legislativo referente al tema de violencia contra la mujer a nivel nacional, estatal e institucional y 2) Los discursos de resistencia que atentan con situar al tema de violencia contra la mujer en el contexto institucional. El análisis del marco legislativo incluyó la revisión a detalle de seis leyes y dos reglamentos de carácter nacional y estatal (3 leyes y 1 reglamento federal y 3 leyes y 1 reglamento de carácter estatal). El marco normativo institucional incluyó la revisión de los dos principales instrumentos normativos (estatuto general y estatuto de alumnos), así como del reglamento para la igualdad de género diseñado por la institución. Para analizar dichos discursos de resistencia a nivel institucional, se revisaron las cifras y descripciones, así como las omisiones y/o vacíos presentes en los informes de actividades que están abiertos al público en general para su consulta y con los cuales las distintas instancias rinden cuentas de su labor a la institución. Específicamente, se revisaron los informes anvales emitidos por: 1) La Defensoría de los Derechos Universitarios (DDU, 2007-2015 y 2017-2018; once documentos en total con extensión variable de 7 a 18 páginas), 2) Centro para el Desarrollo Humano e Integral de los Universitarios (CEnDHIU, 2010-2018; ocho documentos en total con extensión variable de 3 a 12 páginas) y 3) Coordinación de la Unidad de Género (CUG, 2015, 2017-2018; dos documentos en total con extensión de 2 y 6 páginas). En particular, se identificaron los elementos que contribuyen a: 1) normalizar la violencia contra la mujer, 2) desviar la atención para la atribución de responsabilidades y 3) los discursos y las prácticas que legitima la violencia y tienden a distorsionar los actos de violencia perpetuados. Lo anterior se revisó a través del manejo que hizo la institución para responder a las denuncias recibidas, así como la resolución, seguimiento y cumplimiento del dictamen emitido. Adicionalmente, se analizó la presencia y/o ausencia de los indicadores de género reportados y no reportados por las distintas instancias en la institución. Si bien existe una labor e inversión importante en tiempo y recursos dirigidos a difundir, concientizar y capacitar a la comunidad universitaria sobre sus derechos universitarios, salud y prevención de enfermedades (incluidas las de trasmisión sexual); la presente investigación se centra en revisar y evaluar aquellos indicadores asociados a denuncias que se iniciaron para notificar a la institución sobre violaciones y transgresiones a los derechos particulares de los universitarios dentro del contexto institucional. 


\section{Resultados y discusión Marco legislativo a nivel nacional y estatal}

Aprobado por el H. Congreso de la Unión de los Estados Unidos Mexicanos, en 2003 se publicó la Ley Federal para prevenir y eliminar la discriminación (DOF, 2003). La ley homónima se publicó en 2013 para el estado de Veracruz (Gaceta Oficial del Estado de Veracruz, 2013). La ley con carácter federal cuenta con 88 artículos en su versión original y se nombra directamente a la mujer en tres ocasiones. En dos de esas ocasiones se hace referencia a la obligación que tienen los poderes públicos federales y las instituciones para establecer las medidas de nivelación, acceso, permanencia y promoción que garanticen el acceso e igualdad de oportunidades (Artículo 15 Ter; DOF, 2018 y Artículo 15 Octavus; DOF, 2018, respectivamente). La tercera mención declara a el Instituto de la Mujer como dependencia perteneciente al Poder Ejecutivo Federal, el cual debe estar representado ante la Junta de Gobierno (Artículo 23; DOF, 2018). En lo que respecta a la ley homónima para el estado de Veracruz, ésta cuenta con 33 artículos en su versión original. En dicha ley de carácter estatal, se hace mención a la mujer en dos ocasiones. En ambas ocasiones refiere a la obligación de establecer las medidas: 1) compensatorias para eliminar los obstáculos que limitan el ejercicio pleno de libertades y derechos (Artículo 10; Gaceta Oficial del Estado de Veracruz, 2013) y 2) de igualdad de oportunidades y no discriminación asociado al acceso y uso de los medios de transporte público (Artículo 12; DOF, 2018).

En 2006 se publicó la Ley General para la igualdad entre mujeres y hombres (DOF, 2006). La versión homónima para el estado de Veracruz se publicó en 2009 (Gaceta Oficial del Estado de Veracruz, 2009, 2014a). La ley de carácter federal cuenta en su versión original con 49 artículos. En dicha ley se vislumbra a el tema de violencia contra las mujeres o en sentido extenso de violencia de género en seis ocasiones. Primero, plantea la necesidad de erradicar la violencia contra las mujeres (Artículo 17, fracción VII; DOF, 2013). Segundo, reconoce al Sistema Nacional como medio para la modificación de estereotipos que discriminan y fomentan la violencia de género (Artículo 26, fracción III; DOF, 2006). Tercero, plantea la revisión de políticas de prevención, atención, sanción y erradicación de la violencia de género (Artículo 37, fracción III; DOF, 2013). Cuarto, enuncia que la Política Nacional tiene la obligación de erradicar las distintas modalidades de violencia de género (Artículo 39; DOF, 2006). Quinto, reconoce la necesidad de establecer los mecanismos para la atención de las víctimas en todos los tipos de violencia contra las mujeres, incluido el fomento a la investigación en materia de prevención, atención, sanción y erradicación de la violencia contra las mujeres (Artículo 40 fracción IX sic y fracción X sic, respectivamente; DOF, 2013). Sexto, declara la importancia de eliminar los estereotipos que fomentan la discriminación y violencia contra las mujeres como objetivo central en la Política Nacional (Artículo 41, DOF, 2006). En su versión estatal, la Ley para la igualdad entre mujeres y hombres cuenta con 47 artículos en su versión original y hace referencia a la violencia de género en tres ocasiones. Primero, menciona la competencia que tiene el Estado para el desarrollo, implementación y evaluación de los mecanismos para la erradicación de la violencia de género (Artículo 13, fracción V; Gaceta Oficial del Estado de Veracruz, 2009, 2014a). Segundo, establece la 
importancia de desarrollar los lineamientos para la igualdad de género, así como para el establecimiento de las medidas que permitan erradicar toda forma y modalidad de violencia de género (Artículo 17, fracción Vl; Gaceta Oficial del Estado de Veracruz, 2009, 2014a). Tercero, reconoce la obligación que tienen las dependencias y entidades de la Administración Pública estatal y municipal para garantizar el derecho de igualdad entre hombres y mujeres, incluida una vida libre de violencia de género (Artículo 32, fracción V; Gaceta Oficial del Estado de Veracruz ,2009).

En 2007 se publicó la Ley General de acceso de las mujeres a una vida libre de violencia (DOF, 2007). La versión homónima para el estado de Veracruz se publicó en 2008 (Gaceta Oficial del Estado de Veracruz, 2008). A nivel federal, la ley cuenta con 60 artículos en su versión original y en dicha ley se distinguen las distintas modalidades de violencia en el ámbito familiar (3 artículos), ámbito laboral y docente (6 artículos) y en la comunidad (2 artículos). Para el ámbito laboral y docente, 1) se describe a la violencia laboral o docente como un acto $u$ omisión en un solo evento o una serie de eventos relacionados con el abuso de poder, el acoso y hostigamiento sexual que daña la autoestima, salud, libertad, integridad, seguridad y desarrollo de la víctima (Artículo 10; DOF, 2007). 2) Se considera como violencia laboral y/o docente a la negativa ilegal de contratar o respetar la permanencia de la persona en el trabajo o descalificar su trabajo a través de amenazas, humillación, intimidación y explotación (Artículo 11; DOF, 2014a). 3) La violencia docente se refiere a las conductas que dañan la autoestima de las alumnas con actos de discriminación ya sea por su sexo, condición social, edad, situación académica, características físicas y/o por sus limitaciones (Artículo 12; DOF, 2007). 4) El hostigamiento sexual se considera como un ejercicio de poder en una relación de subordinación en ambientes laborales y/o escolares e incluye: conductas verbales, físicas o ambas; o bien las relacionadas con la sexualidad en una connotación lasciva. En este sentido, el acoso sexual se distingue como forma de violencia sin condición de subordinación, pero en referencia a un ejercicio abusivo del poder (Artículo 13; DOF, 2007). 5) Las entidades federativas y el Distrito Federal tienen la atribución para establecer las políticas públicas que fomenten, garanticen, diseñen y promuevan el derecho de las mujeres a una vida libre de violencia (Artículo 14; DOF, 2007). 6) El hostigamiento o acoso sexual es un tema que compete a los tres órdenes de gobierno, por lo que su obligación es reivindicar la dignidad de las víctimas. Por lo anterior, se deben diseñar y establecer los mecanismos para su erradicación en centros laborales y escuelas tanto públicas como privada. Las quejas o denuncias dirigidas al mismo acosador $u$ hostigador deberán tratarse como problema mayor. Paralelamente, se implementarán sanciones administrativas para los superiores jerárquicos que impidan, obstaculicen, omitan, nieguen $u$ oculten la recepción y seguimiento de quejas y/o denuncias de determinado hostigador o acosador (Artículo 15; DOF, 2007). La ley homónima para el estado de Veracruz incluye 46 artículos en su versión original y hace referencia a la violencia laboral y/o escolar en dos ocasiones. Primera, se define a la violencia laboral como una omisión o acto de abuso de poder que daña o lesiona la autoestima, integridad, salud, seguridad y libertad de la víctima. Aunado a esto, describe los actos que reconoce como violatorios tal y como se describen en la ley federal (Artículo 8, fracción III, inciso A; Gaceta Oficial del Estado de Veracruz, 2014b). En el 
mismo artículo se define a la violencia escolar, el hostigamiento sexual y las conductas que dañan la autoestima de las alumnas a manera de actos de discriminación por las mismas características descritas en la ley federal. En adición, considera como forma de violencia escolar la inclusión de imágenes de la mujer con contenidos sexistas en los libros de texto (Artículo 8, fracción III, inciso B; Gaceta Oficial del Estado de Veracruz, 2008). Segunda, hace referencia a la competencia que tienen los gobiernos estatales y municipales para el desarrollo e implementación de políticas públicas que garanticen el derecho de las mujeres y las niñas a una vida libre de violencia en sus relaciones escolares y/o laborales (Artículo 10; Gaceta Oficial del Estado de Veracruz, 2008).

En 2008 se publicó el Reglamento de la Ley General y acceso de las mujeres a una vida libre de violencia (DOF, 2008), mientras que su versión homónima para el estado de Veracruz se publicó en 2010 (Gaceta Oficial del Estado de Veracruz ,2010). El reglamento federal cuenta con 65 artículos en su versión original y aborda la prevención, atención, sanción y erradicación de actos que generan violencia contra las mujeres como ejes de acción (DOF, 2008, 2014b). El reglamento homónimo para el estado de Veracruz cuenta con 87 artículos en su versión original y aborda la prevención, atención, sanción y erradicación de la violencia contra las mujeres como ejes de acción dirigidos al nivel estatal y municipal (Gaceta Oficial del Estado de Veracruz, 2010).

Lo descrito con anterioridad muestra que México cuenta con un marco legislativo dirigido a visibilizar y condenar la violencia contra las mujeres. Lo anterior parte de las acciones dirigidas a prevenir y erradicar la discriminación, así como de la promoción para la igualdad entre mujeres y hombres. No menos importante, la legislación reconoce el derecho que tienen las mujeres para gozar de una vida libre de violencia (DOF, 2003, 2006, 2007, 2008, 2013, 2014a, b; 2018). Para el estado de Veracruz existe un rezago de 1 a 10 años para la promulgación de dicho marco legislativo a nivel estatal (Gaceta Oficial del Estado de Veracruz, 2008, 2009, 2010, 2013, 2014a, b). A pesar de existir dicho marco legislativo, destaca la falta de reconocimiento y mención de la violencia contra la mujer en el ambiente laboral y docente a nivel estatal. Lo anterior refleja una resistencia para nombrar, reconocer y situar que existe ese tipo de violencia dirigido a la mujer y que merece ser tratado como tal y en sus contextos específicos. Específicamente, con base en los informes emitidos por el gobierno federal se sabe que los Poderes Ejecutivo, Legislativo y Judicial, así como la Fiscalía General del Estado de Veracruz incumplen con las condicionantes mínimas requeridas para hacer valer los derechos de las mujeres a una vida libre de violencia a nivel estatal (Informe, 2016, 2017; SEGOB, 2016, 2017).

\section{Marco legislativo a nivel institucional}

El estatuto general y el estatuto de alumnos son los principales instrumentos normativos en la institución. El estatuto general cuenta con 350 artículos, mientras que el estatuto de alumnos cuenta con 179 artículos (Estatuto General, 2013 y Estatuto de alumnos, 2008). En el estatuto 
general universitario se aborda la designación y atribución de la Coordinación de Género y del nombramiento de la persona representante (Artículo 77.1 - 77; Estatuto General, 2013). En dicho documento se enfatiza el compromiso que asume la institución por promover la equidad de género. Dicho compromiso se alinea a las políticas públicas nacionales y estatales. En el documento se manifiesta el compromiso en asesorar, acompañar y seguir aquellos casos relacionados con hostigamiento sexual, acoso sexual, discriminación por sexo o género, así como para atender cualquier tipo de violencia cometida hacia quienes integran la comunidad universitaria (Artículo 77.3, sección VI y sección XIV; Estatuto General, 2013). En el estatuto de los alumnos se alude a la figura de la Defensoría de los Derechos Universitarios como instancia para presentar quejas cuando se consideren que se han violentado los términos y procedimientos establecidos en la normatividad institucional (Artículo 59, Artículo 130 y Artículo 168, Sección V; Estatuto de alumnos, 2008). A este marco normativo referencial se adhiere el Reglamento para la igualdad de género, el cual se incorporó después de ser aprobado por la sesión del H. Consejo Universitario General en 2015 (Reglamento para la igualdad de género, 2015). Dicho documento cuenta con 39 artículos en apoyo a la Coordinación de la Unidad de Género y establece las normas para promover y garantizar la igualdad entre mujeres y hombres, así como para prevenir, atender y erradicar todo tipo de discriminación basada en el sexo o género en la institución. En particular, la perspectiva de género se plantea como tema transversal para incorporase en los programas institucionales y entidades académicas (Artículo 7; Reglamento para la igualdad de género, 2015). Por ello, cada entidad académica debe tener un Representante de Equidad de Género quiénes serán propuestos por directores y designados por Juntas Académicas (Artículo 30; Reglamento para la igualdad de género, 2015). En este sentido, se otorgan atribuciones y responsabilidades a los Representantes de Género de cada entidad para fomentar la equidad e igualdad de género, así como la prevención, atención y erradicación de este tipo de violencia (Artículo 32; Reglamento para la igualdad de género, 2015). En la institución el tema de violencia contra la mujer se circunscribe a los temas de hostigamiento sexual, acoso sexual y discriminación por sexo (Estatuto General, 2013; Reglamento para la igualdad de género, 2015). Por lo anterior, la denuncia constituye un procedimiento por el cual se hace notar a la institución que presuntamente se han violentado y transgredido los derechos de algún miembro de la comunidad universitaria.

La revisión del marco legislativo a nivel institucional muestra que, si bien la institución reconoce el hostigamiento sexual, acoso sexual y discriminación por sexo como parte del problema, la institución no reconoce de forma explícita los actos de violencia contra la mujer en el ámbito laboral y docente. Debido a esto, se presenta un reconocimiento parcial y fragmentado del problema, por lo que la respuesta institucional puede llegar a ser limitada al no estar claramente fundamentada y apoyada por la legislación y normatividad institucional. Esta ausencia y/o vacío normativo contribuye a la normalización de la violencia contra la mujer, minimizando la ocurrencia, frecuencia e incidencia de este tipo de violencia en la comunidad universitaria. 
El diálogo que se establece entre el marco legislativo nacional, estatal e institucional es incompleto. A nivel federal se percibe un avance en el marco legislativo dirigido a visibilizar y condenar la violencia contra la mujer, pero no plantea la forma en cómo abordar el problema ni especifica el tipo de sanciones a los individuos transgresores, así como el acompañamiento y apoyo a las víctimas. Aunado a esto, el avance que pudiera percibirse en visibilizar la violencia contra la mujer se va erosionando con forme la legislación de carácter federal baja a un nivel estatal e institucional.

La falta de interlocución y articulación entre el marco legislativo federal, estatal e institucional dificulta el manejo que se dé a el problema de violencia contra la mujer en los contextos reales. Específicamente, al ser el marco normativo institucional incompleto y fragmentado con respecto al marco normativo federal, la autoridad (institución) puede deslindarse de la responsabilidad de abordar el problema de violencia contra la mujer al argumentar que está fuera de su marco normativo y de su área de competencia. El deslinde de responsabilidades contribuye a normalizar la violencia contra la mujer en la institución.

\section{Discurso de resistencia}

Implementada en 2007, la Defensoría de los derechos Universitarios (DDU) reportó la existencia de 4070 expedientes que abrió de 2007 a 2017. A estas cifras se excluyeron lo acontecido en el 2016, ya que el informe fue inexistente en la base de datos de consulta pública. Aunado a esto, los datos contenidos en el informe de actividades correspondiente al año 2017 y 2018 fueron inconsistentes e incompletos. Independientemente de lo anterior, es notable que el número de quejas, inconformidades y denuncias que ha hecho la comunidad universitaria se incrementó a lo largo del tiempo (de 101 a 589 expedientes de 2007 a 2015). Las actividades de mediación, gestión y conciliación repercutieron en la resolución de la mayor proporción de las quejas e inconformidades descritas en los expedientes (85\%). En segundo orden de importancia se encuentra la proporción de expedientes en los cuales la DDU se declaró como no-competente (3\%). La DDU ha emitió un dictamen con recomendación después de un proceso de investigación y seguimiento de una queja en aproximadamente el $10 \%$ de los casos revisados, mientras que el $2 \%$ de los casos se citó como pendiente de resolución. En cifras, la DDU ha revisado 391 casos (expedientes) y ha valorado que se han transgredido los derechos universitarios dentro de la Institución en 238 casos (61\% de casos dictaminados a favor del demandante), mientras que 153 casos permanecieron como denuncias infundadas (39\% de casos dictaminados como no procedentes). Una vez que la DDU emite un dictamen y/o recomendación a favor del demandante, la autoridad transgresora (docentes, directivos, funcionarios y/o cuerpos colegiados): 1) acepta y cumple (en su totalidad o de forma parcial) con la recomendación emitida por la DDU (82\% de los casos), o 2) rechaza la recomendación, por lo que se incurre en impunidad de la violación y/o transgresión a los derechos de los universitarios (18\% de los casos). En lo que se refiere a los indicadores de género, sólo se aportaron datos para los informes correspondientes al año 2007 y 2009 (48\% mujeres y 52\% hombres en el informe de 2007, 52\% 
mujeres y $48 \%$ hombres en el informe de 2009). Si bien la DDU desempeña un papel fundamental para la orientación, resolución de quejas y deslinde de responsabilidades; la DDU ha omitido indicadores de género que pudieran jugar un papel central en situar el problema dentro de un marco de violencia contra la mujer en la institución. Aunado a esto, destaca el $18 \%$ de impunidad de casos en los cuales la autoridad violentó los derechos universitarios de terceros sin sanción. En un año este indicador se incrementó en un 3\% con respecto al reportado por Hernández-Ramírez (2017).

SibienlaDDUtipificalastransgresionescometidasalacomunidaduniversitaria, laDDUnovincula la ocurrencia, frecuencia, incidenciayreincidencia delosmismossujetosy/o autoridades transgresoras, así como a los sectores y/o personas que son el blanco de agresiones y transgresiones.

Independientemente de los vacíos e inconsistencias detectadas, destaca los cambios positivos que la DDU ha implementado desde el año 2018 en términos de transparencia y rendición de cuentas. A partir de 2018, la DDU pone a disposición del público en general las actas en las cuales se emite un dictamen asociado a un expediente que estuvo sujeto a investigación. En dicho expediente se incluye la descripción del proceso que acompañó la resolución emitida por cada expediente, así como los nombres de las autoridades bajo averiguación y los cargos que ocupa en la institución, mientras que se mantiene el anonimato de las personas que presentaron la queja. Lo anterior representa un avance importante en exponer a las autoridades transgresoras (personas y cargos) y sentar las bases para un estado de derecho real dirigido al respeto de los derechos individuales y colectivos de los universitarios en la institución.

Implementado en 2010, el Centro para el Desarrollo Humano e Integral de los Universitarios (CEnDHIU) centra su labor en los servicios a la salud desde el nivel preventivo y de diagnóstico oportuno, hasta la referencia de casos a médicos especializados. Lo anterior lo hace a través de cursos, pruebas rápidas de VIH (78 - 4356 exámenes realizados), inventarios multifacéticos de personalidad (155 - 222 entrevistas realizadas), estudios psicométricos (16-396 estudios realizados), servicios de orientación vocacional (7 - 24 orientación realizada) y consejería o asesoría (46-61 asesorías realizadas). La entidad aborda temas como la salud reproductiva y sexual (2823 - 7453 beneficiarios; 13562 - 37930 preservativos entregados). En lo que respecta a indicadores, el CEnDHIU solo aportó un indicador por género en su informe de actividades 2011-2012 (atención a 110 alumnos, de los cuales 65\% fueron mujeres y 35\% fueron hombres; CEnDHIU, 2011-2012). El CEnDHIU cuenta con dos Centros Centinela que son unidades de vigilancia de factores de riesgo para la salud, por lo que representa una instancia de acercamiento importante a la comunidad estudiantil. Por sus características, el CEnDHIU cumple una función preventiva y de primer acercamiento en la cual la comunidad estudiantil puede reconocer el fenómeno de violencia contra la mujer. No obstante, se requiere que el CEnDHIU proporcione información clara, precisa y bien sistematizada que pueda ser utilizada como indicadores de riesgo de violencia por género.Implementada en 2014, la Coordinación de la Unidad de Género (CUG) centra su labor en hacer transversal la perspectiva de género en la institución. Por lo anterior, enfatiza su trabajo en la promoción 
de la igualdad de derechos y oportunidades entre hombres y mujeres (equidad de género). En término de cifras, la CUG reportó haber realizado 79 asesorías en el periodo comprendido de 2015 a 2017. El informe de 2016 es inexistente o cuando menos no está disponible para su consulta. El informe 2018 está incompleto y es inconsistente. La CUG reportó una diminución de cerca del $50 \%$ de las asesorías jurídicas realizadas de 2015 a 2016, y una disminución de cerca del $30 \%$ de asesorías jurídicas de 2016 a 2017. La CUG declaró su incompetencia para tratar el $21 \%$ de los casos recibidos en el año 2015. Por otro lado, la CUG reportó que cerca del $50 \%$ de las asesorías jurídicas que otorgó a los universitarios se tradujo en denuncias formales para el año de 2015 y 2017. Dichas asesorías jurídicas siguieron una vía institucional con sanciones impuestas para la mayoría de estos casos. Todos estos casos se asociaron a violencia sexual, incluidos el acoso y hostigamiento sexual, desde tocamientos hasta violaciones (CUG, 2015). Las sanciones que se dictaron se dirigieron en su totalidad a varones, incluidos académicos, estudiantes y trabajadores eventuales. Se menciona que hubo tres casos en los cuales no se dictó sanción por falta de elementos (i.e., denuncia infundada).

La presencia de la CUG abre un espacio en la institución para nombrar, tratar y prevenir la discriminación y los actos de violencia asociado al género y en particular de violencia contra la mujer. No obstante, destaca la disminución en el número de asesorías jurídicas reportadas. Con base en los informes de actividades sólo se trataron denuncias formales para los años de 2015 y 2017. Lo anterior puede estar relacionado con las acciones de escrutinio a la que se exponen las mujeres universitarias y que tienen que demostrar actos de acoso, hostigamiento sexual y violación. En este sentido, varios autores reconocen que los procesos administrativos diseñados para tratar este tipo de denuncias en las IES suelen obstaculizar de manera sistémica el acceso que pudieran tener las mujeres a los mecanismos de protección y justicia (Koss, 2000; Cantalupo, 2012; Mingo y Moreno, 2015). Específicamente, los actos de escrutinio parten de la premisa en la cual se debe descartar que la víctima tuvo la culpa o propició la situación de violencia, por lo que las mujeres deben transitar por procesos desgastantes y muchas veces degradantes para comprobar que sus derechos fundamentales fueron violentados (Berns, 2001; Mengo y Black, 2015; Barreto, 2017).

La Universidad Veracruzana cuenta con 130 entidades académicas distribuidas en las cinco regiones del estado de Veracruz, las cuales incluyen facultades, institutos y centros de Investigación como entidades académicas. A este número, se suman 16 entidades académicas adscritas a la institución con un carácter más bien transversal, pero con sedes específicas a nivel regional. Lo anterior excluye a las cuatro vicerrectorías localizadas en la región de Veracruz-Boca del Río, Coatzacoalcos-Minatitlán, Orizaba-Córdoba y Poza Rica-Tuxpan. En términos generales, la institución reporta a un total de 70 Representantes de Género $177 \%$ mujeres y $23 \%$ hombres) para cubrir un requerimiento normativo y legal establecido en 2013 de cuando menos 146 entidades académicas adscritas a la universidad. Es decir, la institución cumple con el $48 \%$ de las figuras legales y administrativas que cumplen con la función de Representantes de Género en su entidad académica en concordancia con la legislación y normatividad institucional (Estatuto General, 2013; Reglamento para la igualdad de género, 2015). Cabe señalar, que los Representantes de Género deben ser propuestos por 
Directivos y designados por Juntas Académicas (Artículo 30; Reglamento para la igualdad de género, 2015). No obstante, existe una renuencia y retraso de cinco años para que el $52 \%$ de las entidades académicas de la institución asigne a su Representante de Género. En cifras, es evidente observar una sobrerrepresentación de mujeres con respecto a los hombres que cumplen el cargo de Representantes de Género en la Institución $177 \%$ mujeres y $23 \%$ hombres). No obstante, esta cifra se contradice cuando se reconoce el predominio de los hombres en los cargos directivos cuando se revisa a detalle la organización de las entidades académicas. Específicamente, un análisis preliminar realizado por entidad académica adscrita al Área Biológico-Agropecuaria de la Región de Xalapa, muestra un predominio de los hombres que ocupan un puesto de autoridad (puesto de poder) en cinco entidades. En contraparte, las mujeres ocupan un puesto de autoridad (puesto de poder) por encima de los hombres en dos entidades, mientras que en dos entidades prevalece el principio de paridad tal y como se puede observar en la Tabla 1.

Tabla 1:

Relación del número de mujeres y del número de hombres que ocupan un puesto dirigente por entidad académica adscrita al Área Biológico-Agropecuaria Región Xalapa de la Universidad Veracruzana. Puesto de Poder incluye: directores o coordinadores, secretaria académica, coordinación de posgrado, coordinación de sustentabilidad.

\begin{tabular}{ccccc}
\hline Entidad & Puesto de poder & Consejo Técnico & $\begin{array}{c}\text { Representante de } \\
\text { Género }\end{array}$ & $\begin{array}{c}\text { Número total de } \\
\text { académicos }\end{array}$ \\
\hline $\begin{array}{c}\text { Facultad de Biología } \\
\begin{array}{c}\text { Facultad de Ciencias } \\
\text { Agrícolas }\end{array}\end{array}$ & $2: 2$ & $3: 4$ & 0 & $21: 37$ \\
$\begin{array}{c}\text { Instituto de Neuro eto- } \\
\text { logía }\end{array}$ & $1: 2$ & $2: 4$ & 0 & $15: 40$ \\
$\begin{array}{c}\text { Instituto de Investigacio- } \\
\text { nes Biológicas }\end{array}$ & $1: 1$ & $2: 5$ & 0 & $6: 5$ \\
$\begin{array}{c}\text { Instituto de Investigacio- } \\
\text { nes Forestales }\end{array}$ & $4: 0$ & $0: 5$ & 0 & $6: 12$ \\
$\begin{array}{c}\text { Instituto de Biotecnología } \\
\text { y Ecología Aplicada }\end{array}$ & $0: 2$ & N/D & 0 & $9: 13$ \\
$\begin{array}{c}\text { Centro de Investigacio- } \\
\text { nes Tropicales }\end{array}$ & $3: 1$ & $2: 5$ & 0 & $5: 13$ \\
$\begin{array}{c}\text { Centro de Eco alfabe- } \\
\text { tización y Diálogo de } \\
\text { Saberes }\end{array}$ & $0: 4$ & $4: 3$ & 1 (mujer) & $9: 11$ \\
$\begin{array}{c}\text { Centro de Investigacio- } \\
\text { nes Biomédicas }\end{array}$ & $0: 2$ & $2: 5$ & 0 & $6: 7$ \\
\hline
\end{tabular}

Fuente: Elaboración propia a partir de la información reportada por cada entidad académica revisada.

A nivel de Consejo Técnico, el número de hombres sobre pasa al número de mujeres en siete de nueve entidades con base en la información mostrada en la Tabla 1. En la Tabla 1 se observa que, en ocho de nueve entidades, el número de hombres sobre pasa al número de mujeres adscritas a su entidad y solo una entidad posee Representante de Género. Si 
bien, algunas diferencias pueden no ser acentuadas, existen casos en los cuales no figura la presencia de la mujer en puesto de autoridad (cinco casos evaluados) y en un caso no figura la presencia de los hombres en algún puesto de autoridad. Lo anterior ejemplifica una estructura jerárquica y simbólica desigual entre hombres y mujeres, lo que hace real y sustantiva la inequidad de género vista desde la distribución desigual del poder. Esta realidad a su vez transgrede el principio constitucional de paridad e igualdad, que debería predominar a nivel institucional.

La violencia contra la mujer es un tema de interés y de urgencia dadas las denuncias públicas recibidas en los últimos años en la Universidad Veracruzana, por lo que la institución ha alineado su legislación y normatividad a las propuestas legislativas de carácter federal y estatal. De manera coordinada, la Universidad Veracruzana ha centrado sus programas académicos y estrategias de trabajo para hacer de la equidad de género un tema transversal a nivel institucional (Programa de Trabajo Estratégico, 2017-2021). La DDH, el CEnDHIU y la CUG constituyen instancias de apoyo para informar, prevenir y procurar el respeto a los derechos que la normatividad y la legislación universitaria otorga a los miembros de la comunidad universitaria. No obstante, los esfuerzos de la institución para prevenir, tratar y erradicar la violencia contra la mujer en el ambiente universitario se enfrentan a un discurso de resistencia. Específicamente, la violencia contra la mujer en la institución se normaliza a través de: 1) proteger las jerarquías de privilegio y de desigualdad (principio de paridad no respetado), 2) permitir que prevalezca la impunidad en casos que fueron investigados y dictaminados como culposos (Jordan, Combs y Smith, 2014; Hernández-Ramírez, 2017), 3) descartar que las transgresiones pueden ser eventos repetitivos y no aislados, por lo que la institución no reconoce la reincidencia delictiva de una persona y/o sobre un mismo sector vulnerado (i.e., mujeres, estudiantes, etc., Hernández-Ramírez, 2017) y 4) ocultar informes o bien presentarlos de forma inconsistente y/o incompletos (Jordan, Combs y Smith, 2014; Hernández-Ramírez, 2017).

La institución incurre en un desvío de la atención al tema de violencia contra la mujer a través de: 1) no situar el problema en un marco de referencia de violencia contra la mujer; en su lugar reconoce y dictamina la problemática a tratar en términos de problemas en la comunidad universitaria (Estatuto General, 2013 y Estatuto de alumnos, 2008), 2) prevalece la omisión en reportar indicadores de género en prácticamente el $88 \%$ de los informes emitidos por las distintas instancias revisadas (DDU, 2007-2018; CEnDHIU, 2010-2018; CUG, 2017-2018).

La institución legitima la violencia a través de: 1) la prevalencia en la impunidad de casos que fueron evaluados y dictaminados como culposos y en los cuales la autoridad responsable rechazó el dictamen sin recibir algún tipo de sanción (DDU, 2007-2018; CEnDHIU, 20102018; CUG, 2017-2018; Hernández-Ramírez, 2017), 2) la declaración de incompetencia por tener un marco legislativo y normativo limitado, justifica y deslinda la responsabilidad que tiene la institución a través de sus diferentes instancias para abordar y atender las problemáticas que ocurren en la comunidad universitaria. Por lo anterior, prevalecen y se repiten este tipo de transgresiones a nivel institucional (DDU, 2007-2018; CEnDHIU, 2010-2018; CUG, 
2017-2018; Hernández-Ramírez, 2017) y 3) la falta de atención para no reconocer otras modalidades de violencia contra la mujer que están descritas para el ámbito laboral y docente en la legislación federal pero que no se han incorporado a la normatividad institucional propicia que se legitime la violencia contra la mujer en la institución (DOF, 2007).

La violencia contra la mujer se arraiga en los patrones de desigualdad, subordinación y de asimetrías en el ejercicio del poder (Hernández-Ramírez, 2017; Instituto Nacional de las Mujeres, 2019). En este sentido, la violencia contra la mujer se enfrenta a una carga histórica de privilegios hacia el género masculino, de ahí la persistencia en el discurso de resistencia.

\section{Conclusiones}

La violencia contra la mujer se arraiga en la distribución desigual de las jerarquías de poder de orden simbólico y del ejercicio desigual de los derechos asociados al género en las IES. El ciclo de normalización de la violencia, el desvío de la atención, la distorsión de hechos y la deficiencia e impunidad de los procesos de denuncia debe romperse en beneficio de la construcción de sociedades justas e incluyentes. Los datos no disponibles asociados con la custodia de indicadores de la información (i.e., quién mantiene la información y otorga acceso a ese conjunto de información) es otra forma de negación para reconocer la violencia.

Las oportunidades para hacer real y sustantiva los derechos de las mujeres y de los hombres en la Universidad Veracruzana requiere reforzar y mejorar los procesos que acompañan las denuncias, los procesos de escrutinio y sanción para evitar la impunidad. De manera paralela, se debe mejorar el registro, sistematización y los procesos de rendición de cuentas y transparencia de los indicadores generados. Lo anterior permitirá evaluar cualitativa y cuantitativamente los procedimientos aplicados y realizar planes de mejora con base en las experiencias generadas y en beneficio de la comunidad universitaria. El presente trabajo representa un llamado de atención a la institución para que se sitúe y reconozca el problema de violencia contra la mujer en su contexto universitario. Este llamado busca propiciar la responsabilidad y obligatoriedad que tiene la institución para enfrentar dicho problema, así como de otorgar información sistematizada, pública, confiable, verificable y transparente dirigida a mejorar el aprendizaje institucional. Es obligación de la institución atender la problemática de violencia contra la mujer desde la prevención hasta la atención y acompañamiento. El empoderamiento de las mujeres y la política de "cero tolerancias" hacia la violencia contra la mujer constituye una estrategia para enfrentar los discursos de resistencia que atentan cono reconocer, situar y tratar este problema en las IES.

\section{Agradecimientos}

A Carina Saldivia-Rivera y dos revisores anónimos por sus valiosos comentarios que contribuyeron a mejorar substancialmente la presentación de este trabajo. 


\section{Bibliografía}

Barreto, M. (2017). Violencia de género y denuncia pública en la universidad. Revista Mexicana de Sociología, (79), 261-286.

Berns, N. (2001). Degendering the problema and gendering the blame: political discourse on women and violence. Gender and Society, (15), 262-281.

Cantalupo, N.C. (2012). "Decriminalizing" campus institutional responses to sexual assault. Journal of Collage and University Law, (38), 483-526.

CEnDHIU. (2010-2018). Informe de Actividades. Universidad Veracruzana. Recuperado de: https://www.uv.mx/cendhiu/

CUG. (2015). Informe de Actividades. Universidad Veracruzana. Recuperado de: https://www.uv.mx/uge/

. (2017-2018). Informe de Actividades. Universidad Veracruzana. Recuperado de: https://www.uv.mx/uge/

DDU. (2007-2015). Informe de Actividades. Universidad Veracruzana. Recuperado de: https://www.uv.mx/defensoria/

(2017-2018). Informe de Actividades. Universidad Veracruzana. Recuperado de: https://www.uv.mx/defensoria/

DeKeseredy, W.S. (2000). Current controversies on defining nonlethal violence against women in intimate heterosexual relationships. Empirical implications. Violence against women, (6), 728-746.

DOF. (2003). Ley Federal para prevenir y eliminar la discriminación. Cámara de Diputados del H. Congreso de la Unión. Secretaría General. Secretaría de Servicios Parlamentarios. Recuperado de: https://dof.gob.mx/

- (2006). Ley Federal para la igualdad entre mujeres y hombres. Cámara de Diputados del H. Congreso de la Unión. Secretaría General. Secretaría de Servicios Parlamentarios. Recuperado de: https://dof.gob.mx/

(2007). Ley General de acceso de las mujeres a una vida libre de violencia. Cámara de Diputados del H. Congreso de la Unión. Secretaría General. Secretaría de Servicios Parlamentarios. Recuperado de: https://dof.gob.mx/

(2008). Reglamento de la Ley General de acceso de las mujeres a una vida libre de violencia. Cámara de Diputados del H. Congreso de la Unión. Secretaría General. Secretaría de Servicios Parlamentarios. Recuperado de: https://dof.gob.mx/

(2013). Ley Federal para la igualdad entre mujeres y hombres. Última reforma publicada. Cámara de Diputados del H. Congreso de la Unión. Secretaría General. Secretaría de Servicios Parlamentarios. Recuperado de: https://dof. gob.mx/

- (2014a). Ley General de acceso de las mujeres a una vida libre de violencia. Última reforma publicada. Cámara de Diputados del H. Congreso de la Unión. Secretaría General. Secretaría de Servicios Parlamentarios. Recuperado de: https://dof.gob.mx/

- (2014b). Reglamento de la Ley General de acceso de las mujeres a una vida libre de violencia. Última reforma publicada. Cámara de Diputados del H. Congreso de la Unión. Secretaría General. Secretaría de Servicios Parlamentarios. Recuperado de: https://dof.gob.mx/

(2018). Ley Federal para prevenir y eliminar la discriminación. Última reforma publicada. Cámara de Diputados del H. Congreso de la Unión. Secretaría General. Secretaría de Servicios Parlamentarios. Recuperado de: https://dof. gob.mx/

Estatuto de los alumnos. (2008). Legislación Universitaria. Universidad Veracruzana. Recuperado de: https://www.uv.mx/

Estatuto General. (2013). Legislación Universitaria. Universidad Veracruzana. Recuperado de: https://www.uv.mx/

Gaceta Oficial del Estado de Veracruz. (2008). Ley de acceso de las mujeres a una vida libre de violencia para el Estado de Veracruz de Ignacio de la Llave. Órgano del Gobierno del Estado de Veracruz. Recuperado de: http://www. ordenjuridico.gob.mx/Documentos/Estatal/Veracruz/

- (2009). Ley para la igualdad entre mujeres y hombres para el Estado de Veracruz de Ignacio de la Llave. Órgano del Gobierno del Estado de Veracruz. Recuperado de: http://www.ordenjuridico.gob.mx/Documentos/Estatal/Ve- 
racruz/

(2010). Reglamento de la Ley de acceso de las mujeres a una vida libre de violencia para el Estado de Veracruz de Ignacio de la Llave. Órgano del Gobierno del Estado de Veracruz. Recuperado de: http://www.ordenjuridico. gob.mx/Documentos/Estatal/Veracruz/

(2013). Ley para prevenir y eliminar la discriminación en el Estado de Veracruz de Ignacio de la Llave. Órgano del Gobierno del Estado de Veracruz. Recuperado de: http://www.ordenjuridico.gob.mx/Documentos/Estatal/Veracruzl

(2014a). Ley para la igualdad entre mujeres y hombres para el Estado de Veracruz de lgnacio de la Llave. Última Reforma publicada. Órgano del Gobierno del Estado de Veracruz. Recuperado de: http://www.ordenjuridico.gob. $\mathrm{mx} /$ Documentos/Estatal/Veracruz/

(2014b). Ley de acceso de las mujeres a una vida libre de violencia para el Estado de Veracruz de Ignacio de la Llave. Última Reforma publicada. Órgano del Gobierno del Estado de Veracruz. Recuperado de: http://www.ordenjuridico.gob.mx/Documentos/Estatal/Veracruz/

García-Moreno, C., Zimmerman, C., Morris-Gehring, A., Heise, L., Amni, A., Abrahams, N., Montoya, O., Bhate-Doesthali, P., Kilonzo, N. y Watts, C. (2014). Addressing violence against woman: a call to action. The Lancet, (385), 16851695.

Hernández-Ramírez, A.M. (2017). Defensoría de los Derechos Universitarios: revisión y sistematización de su labor de servicio en la universidad. Revista Electrónica de la Coordinación Universitaria de Observatorios de la Universidad Veracruzana UVServa, (3), 69-74.

Huerta. M., Cortina, L.M., Pang, J.S., Torges, C.M. y Magley, V.J. (2006). Sex and power in the academy: Modeling sexual harassment in the lives of collage women. Personality and Social Psychology Bulletin, (32), 616-628.

INEGI. (2014). Recopilación de información de los organismos públicos de protección y defensa de los derechos humanos en México. Instituto Nacional de Estadística y Geografía, México.

(2015). Censo Nacional de Gobiernos Municipales y Delegacionales 2015. Censo Nacional de Gobierno, Seguridad Pública y Sistema Penitenciario Estatales 2015. Instituto Nacional de Estadística y Geografía, México.

(2018). Encuesta Nacional de Victimización y Percepción sobre Seguridad Pública (ENVIPE). Instituto Nacional de Estadística y Geografía, México.

. (2019). Encuesta Nacional de Victimización y Percepción sobre Seguridad Pública. Instituto Nacional de Estadística y Geografía, México.

Informe. (2016). Dictamen sobre la implementación de las propuestas contenidas en las conclusiones del informe emitido por el grupo de trabajo conformado para atender la solicitud de alerta de violencia de género contra las mujeres en el estado de Veracruz. Comisión Nacional para Prevenir y Erradicar la Violencia contra las Mujeres (CONAVIM).

(2017). Dictamen sobre la implementación de las propuestas contenidas en las conclusiones del informe emitido por el grupo de trabajo conformado para atender la solicitud de alerta de violencia de género contra las mujeres por agravio comparado para el estado de Veracruz. Comisión Nacional para Prevenir y Erradicar la Violencia contra las Mujeres (CONAVIM).

Instituto Nacional de las Mujeres. (2019). Vida sin violencia. Gobierno de México. https://www.gob.mx/inmujeres

Jordan, C.E., Combs, J.L. y Smith, G.T. (2014). An exploration of sexual victimization and academic performance among collage women. Office for Policy Studies on Violence Against Women Publications, (38). https://uknowledge.uky.edu/ ipsvaw facpub/38

Koss, M.P. (2000). Blame, shame, and community: Justice responses to violence against women. American Psychologist, (55), 1332-1343.

Mengo, C. y Black, B.M. (2015). Violence victimization on a collage campus: impact in GPA and school dropout. Journal of Collage Student Retention: Research, Theory and Practice, $1-5$.

Mingo, A. y Moreno, H. (2015). El ocioso intento de tapar el sol con un dedo: violencia de género en la Universidad. Perfiles Educativos, (37), 138-155.

Programa de Trabajo Estratégico. (2017-2021). Plan General de Desarrollo 2030. Uni- 
versidad Veracruzana.

Reglamento para la igualdad de género (2015). Legislación Universitaria. Universidad Veracruzana. Recuperado de: https://www.uv.mx/

SEGOB. (2016). Declaratoria de Alerta de Violencia de Género contra las mujeres Estado de Veracruz. Secretaría de Gobernación, México, D.F.

. (2017). Declaratoria de Alerta de Violencia de Género contra las mujeres por agravio comparado, para el estado de Veracruz. Secretaría de Gobernación, México. D.F.

Tedeschi, J.T. y Felson, R.B. (1994). Violence, aggression \& coercive actions. Washington D.C. United States. American Psychological Association.

van Roosmalen, E.,y McDaniel, SA. (1998). Sexual harassment in academia: a hazard to women's health. Women Health, (28), 33-54.

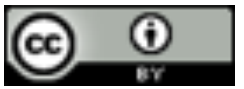

Este es un artículo de acceso abierto bajo licencia Creative Commons Reconocimiento 4.0 Internacional 